\section{Diabetic macular oedema: pilot randomised trial of pars plana vitrectomy vs macular argon photocoagulation}

Jl Patel ${ }^{1,2}$, PG Hykin'1, M Schadt'1, V Luong ${ }^{2}$, C Bunce', F Fitzke ${ }^{2}$ and ZJ Gregor ${ }^{1}$

\begin{abstract}
Introduction Focal macular photocoagulation for clinically significant macular oedema (CSME) is the proven method for treatment of this condition, but with little chance of visual improvement. Pars plana vitrectomy (PPV) may produce resolution of macular oedema and improvement in visual acuity. However, there have been no randomised trials to ascertain role of vitrectomy in the management of persistent CSME.
\end{abstract}

Methods Patients with persistent CSME despite previous macular photocoagulation and Snellen visual acuity $6 / 15$ to $6 / 60$ were recruited. Dilated fundoscopy, best-corrected visual acuity including Early Treatment Diabetic Retinopathy Study (ETDRS) vision, ocular coherence tomography and fundus fluorescein angiography (FFA) at baseline and up to 12 months post-treatment was performed. Exclusion criteria were signs of posterior vitreous detachment, macular traction or the taut posterior hyaloid face syndrome, or macular ischaemia on FFA. In all, 20 patients were randomised (10 in each arm) to either standard macular photocoagulation or PPV and removal of the posterior hyaloid face.

Results Of the 20 patients recruited, seven patients completed the protocol in the vitrectomy and eight in the laser arms, respectively. There was little evidence of any difference in the foveal thickness at $\mathbf{1 2}$ months between the two treatment arms despite a gradual improvement. Only one patient, from the vitrectomy arm, suffered moderate visual loss (defined as loss of 15 ETDRS letters) (our primary outcome).
Discussion In this pilot RCT, standard PPV provides little visual benefit compared to macular photocoagulation, but a larger definitive study is required to confirm this early appraisal.

Eye (2006) 20, 873-881. doi:10.1038/sj.eye.6702012; published onine 29 July 2005

Keywords: diabetic macular oedema; laser; vitrectomy; RCT

\section{Introduction}

The Early Treatment Diabetic Retinopathy Study (ETDRS) showed that focal argon macular photocoagulation can reduce the risk of moderate visual loss (MVL) (loss of 15 ETDRS letters or three lines on ETDRS chart) by 50\% in patients with clinically significant macular oedema (CSME). Consequently, macular laser treatment became the standard approach to the treatment of CSME. ${ }^{1}$

However, $15 \%$ of eyes experienced MVL after 3 years of follow-up despite laser treatment. A quarter of patients with diffuse macular oedema experience visual loss over a 3-year period ${ }^{2}$ and the prognosis after laser treatment is worse in diffuse macular oedema than in the focal type. ${ }^{3}$ Recent studies have suggested that pars plana vitrectomy (PPV) for diffuse macular oedema with either a taut posterior hyaloid face ${ }^{4}$ or with a clinically normal appearing hyaloid face ${ }^{5}$ can lead to improvement in both vision and in macula oedema.

Optical coherence tomography (OCT) in diabetic macular oedema has suggested two types of macular profiles: a dome-shaped group with a thickened retina, signs of vitreomacular
${ }^{1}$ Moorfields Eye Hospital, London, UK

2Department of Pathology, Institute of Ophthalmology, London, UK

Correspondence: JI Patel, Department of Pathology, Institute of Ophthalmology, 11-43 Bath Street, London EC1V 9EL, UK

Tel: + 44207608 6808;

Fax: + 442076086862 .

E-mail: jigs37@

hotmail.com

Received: 26 November 2004

Accepted: 15 February 2005 Published onine: 29 July 2005

Financial disclosure: None 
traction and a large central cyst. The second type was retinal thickening with reduced intraretinal reflectivity and multiple intraretinal cysts. ${ }^{6,7}$ The difference in the anatomical profiles between the two groups would suggest greater vitreomacular tractional forces contributing to macular oedema in the dome-shaped group with the preretinal separation of the posterior hyaloid cortex and persistent attachment to the fovea.

However, data regarding the effectiveness of PPV are limited to mostly retrospective studies and no controlled prospective randomised studies comparing PPV with standard ETDRS macular laser treatment exist. In preparation for such a study, we designed a prospective randomised controlled one-centre pilot trial to provide preliminary data to evaluate the feasibility and assist in the design of a larger definitive trial.

\section{Patients and methods}

Patients with persistent CSME despite previous macular laser were offered enrolment. Each patient gave written consent, using a consent form approved by the local institutional review board and was randomly assigned to the surgery or to the laser arm of the study.

A masked observer assessed the best-corrected visual acuity (BCVA) for both Snellen and ETDRS vision score. All patients underwent dilated retinal examination, fuds intravenous fluorescein angiography (FFA), OCT and fine matrix mapping (FMM). The examinations were performed within 1 month prior to surgery to obtain the baseline clinical parameters and at 2, 6, 12 and 24, and 48 weeks postoperatively. These outcome assessments were made by the same researcher (JP).

Inclusion criteria were (i) persistent CSME involving the foveal centre for less than 2 years, (ii) previous treatment with macular laser, and (iii) ETDRS vision score of 65-35 (equivalent Snellen visual acuity 6/15 to 6/60). Exclusion criteria were: (i) posterior vitreous detachment diagnosed by the presence of a Weiss ring, (ii) macular traction as evidenced by retinal striae involving the foveal centre or the taut vitreous face syndrome, (iii) macular ischaemia as defined by an enlarged foveolar avascular zone (foveolar avascular zone $(\mathrm{FAZ})>1000 \mu \mathrm{m})$ or significant perifoveal capillary loss on FFA, and (iv) coexistent ocular disease.

OCT is a noninvasive, noncontact imaging modality producing high-resolution cross-sectional tomographs of ocular tissue. ${ }^{8}$ It produces a two-dimensional false colour image of the back-scattered light from different layers of the retina analogous to ultrasonic B-scan imaging. The only difference is that optical reflectivity is measured. Axial resolution up to $14 \mu \mathrm{m}$ is achievable. Radial scans (6 mm diameter) centred on the point of fixation were taken at each time point, and assessed qualitatively and analysed quantitatively using the retinal mapping software. Quantitative assessment using the computer software (Zeiss-Humphrey, Dublin, CA, USA, software version A5, using model OCT 2) included an estimation of the foveal thickness and the macular volume, which is derived by an integration process of the average retinal thickness of the six radial scans.

FMM is a psychophysical method of examination, which determines the cone thresholds in a 9 by 9 degree field centred on the point of fixation. In all, 100 points within this test field are analysed using a modified Humphrey field analyser. A three-dimensional representation of photopic white stimulus thresholds (given as log units) can be generated from these data. The lower the threshold, the better the cone function.

Patients were recruited from the Medical Retina Clinics at Moorfields Eye Hospital and randomised by the Statistical Department of Moorfields Eye Hospital in a 1:1 ratio into either PPV arm or the standard ETDRS argon photocoagulation (laser) arm. Only one eye of each patient could be enrolled in the pilot trial. Where both eyes of a patient were eligible, the eye 'chosen' was based on the toss of a coin. The nonselected eye then underwent standard laser treatment.

Patients randomised to PPV underwent standard three-port vitrectomy with elevation and the removal of the posterior vitreous cortex without peeling of the internal limiting membrane (ILM). Fluid- $\mathrm{SF}_{6}$ gas exchange was performed if retinal breaks were found on the $360^{\circ}$ examination of the peripheral retina prior to the conclusion of the operation. Such breaks were treated with laser photocoagulation or cyotherapy.

Subconjunctival injection of Bethamethasone and Cefuroxime was given at the conclusion of the operation. Patients were treated with topical Atropine 1\%, Dexamethasone and Chloramphenicol for 3 weeks after the operation. Patients randomised to laser underwent standard ETDRS argon photocoagulation after clinical and FFA review.

The main goal of this analysis was to determine the number of patients suffering MVL 12 months after treatment. Secondary parameters used were changes in the functional and structural indices 12 months after treatment and the percentage success rate at each time point defined prospectively as either an improvement or no change of more than one-line loss of vision (or five letters on ETDRS chart).

All patients were recruited in accordance with the Declaration of Helsinki and with the approval of the Ethics Committee of Moorfields Eye

Hospital. 


\section{Statistical analysis}

As this was a feasibility study, we primarily conducted a descriptive analysis only. However, given the nature of our results, we explored the data further using analysis of variance as a post hoc analysis to examine evidence of treatment effect on the ETDRS vision at 12 months.

\section{Results}

In all, 20 patients were equally randomised into each treatment arm. Seven patients in PPV arm completed the trial protocol. Three patients failed to have treatment (one was involved in a serious road traffic accident prior to operation date and one had corrected visual acuity better than inclusion criteria and one died prior to treatment). Eight patients in the laser arm completed the trial protocol. One patient was lost to follow-up, and one failed to have treatment (Figure 1). No complications occurred in either treatment arm in any patient.

The demographics, the medical and ocular status including the average duration of macular oedema, and the number of laser treatments prior to recruitment to the study are described in Table 1 for the patients treated in both arms of the study. There was no significant difference in the distribution of factors such as sex and on insulin treatment $(P=0.3147$ Fisher's exact test) between treatment groups. No patient showed significant haematological, cardiac, or renal abnormalities.

Patients who were enrolled in this pilot trial adhered to the examination schedule. Of a total of 32 examinations expected for patients in the laser treatment arm from 6 weeks to 12 months after treatment, 29 (90\%) were completed. Similarly for the PPV group of a total of 28 examinations expected over the same time period, 25 (89\%) were completed.

The patients in the laser-treated arm showed a variable number of macular laser scars of varying intensity, but prior to treatment in the trial, they underwent FFA to evaluate the areas of fluorescein leakage and ischaemia other than the FAZ. On consultation with the Medical Retina consultant $(\mathrm{PH})$, laser treatment was planned for these areas at a power sufficient to achieve greying of the retina. As each patient in this arm showed different patterns and extent of area of leakage and ischaemia, they received different numbers of laser burns.

Table 1 Summary of the demographic and clinical profile of the patients recruited into each arm

\begin{tabular}{|c|c|c|}
\hline & Laser arm & Vitrectomy arm \\
\hline Number & 8 & 7 \\
\hline $\begin{array}{l}\text { Average age } \\
\text { (years) }\end{array}$ & 64 (range 50-71) & 65 (range 61-74) \\
\hline Sex & $\begin{array}{l}\text { Two male; } \\
\text { six female }\end{array}$ & Four male; 5 female \\
\hline Diabetic type & Type 2 & Type 2 \\
\hline $\begin{array}{l}\text { Average years } \\
\text { diabetes }\end{array}$ & 14 (range 5-22) & 15 (range 6-24) \\
\hline HbA1C (\%) & 9 (range $9.5-11 \%$ ) & 9.1 (range $8-10 \%$ ) \\
\hline Hypertension & 5 & 8 \\
\hline $\begin{array}{l}\text { Diabetic } \\
\text { retinopathy }\end{array}$ & $\begin{array}{l}\text { Six moderate-severe } \\
\text { nonproliferative; } 1 \\
\text { quiescent } \\
\text { proliferative }\end{array}$ & $\begin{array}{l}\text { All moderate-severe } \\
\text { nonproliferative }\end{array}$ \\
\hline $\begin{array}{l}\text { Average number } \\
\text { macular laser } \\
\text { treatments }\end{array}$ & 3 (range $1-5$ ) & 3 (range $2-5$ ) \\
\hline $\begin{array}{l}\text { Average duration } \\
\text { of oedema prior to } \\
\text { recruitment } \\
\text { (months) }\end{array}$ & 14 (range 12-18) & 14 (range 12-18) \\
\hline
\end{tabular}

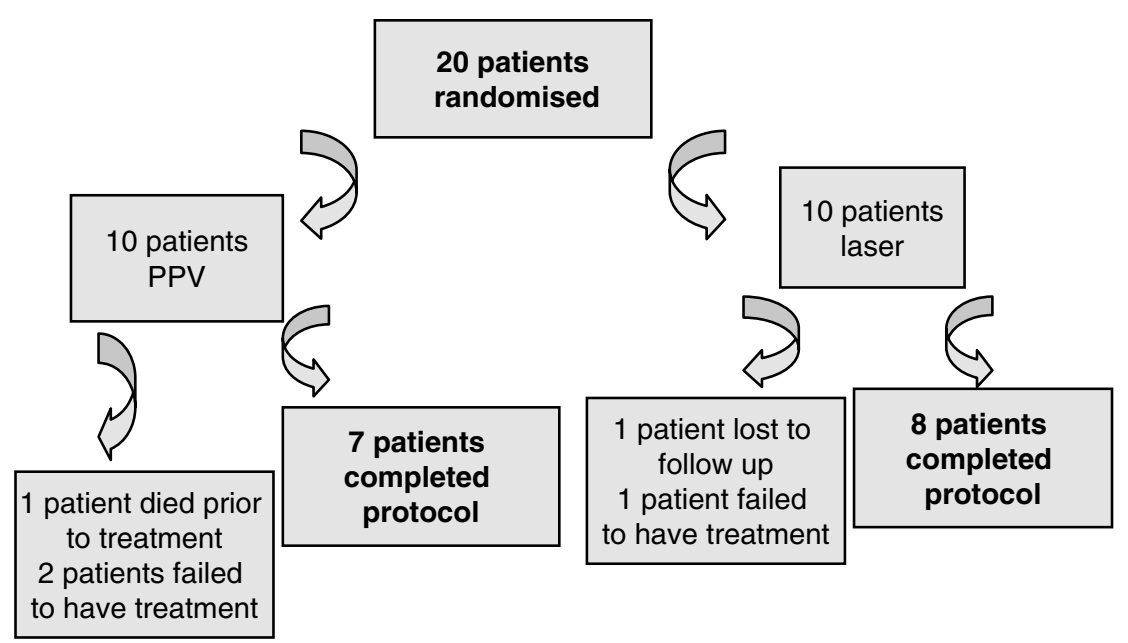

Figure 1 Flow chart depicting the course of the patients in the trial. 


\section{Visual acuity results}

The median baseline ETDRS letters read in the laser group was 62.5 (range 42-75) (Snellen equivalence 6/15, range $6 / 15$ to $6 / 45$ ). At 6 weeks it was 65 (range 50 to 79 ) (Snellen 6/15 to 6/60). At 3 months it had increased to 67 (range 53-79) (Snellen 6/15, range 6/12 to 6/30), at 6 months 66 (range 39-76) (Snellen 6/12, range 6/9 to $6 / 60$ ) and at 12 months post-treatment it was 67.5 (range 61-80) (Snellen 6/12, range 6/9 to $6 / 18$ ). No patients in the laser group suffered from MVL at the 12-month final visit (ie loss of 15 letters on the ETDRS chart) (Figure 2 and Table 2).

The median baseline ETDRS letters read in the PPV group was 58 (range 41-71) (Snellen 6/24, range 6/15 to $6 / 60$ ). At 6 weeks, it was 52 (range 48-68) (Snellen 6/30, range $6 / 12$ to $6 / 30$ ). At 3 months it was 60 (range $46-70$ ) (Snellen $6 / 15$, range $6 / 12$ to $6 / 45$ ), while at 6 and 12 months it was 60 (range 40-74) (Snellen 6/15, range 6/12 to $6 / 45)$. One patient in the PPV group suffered from MVL at the 12-month final visit (Figure 2 and Table 2).

Comparing the vision in the two groups as a post hoc analysis using analysis of covariance, there was no significant difference in the vision between the two groups except at 12 months post-treatment in which the laser group showed a slight but significant improvement in ETDRS vision compared to the PPV group $(P=0.03)$.

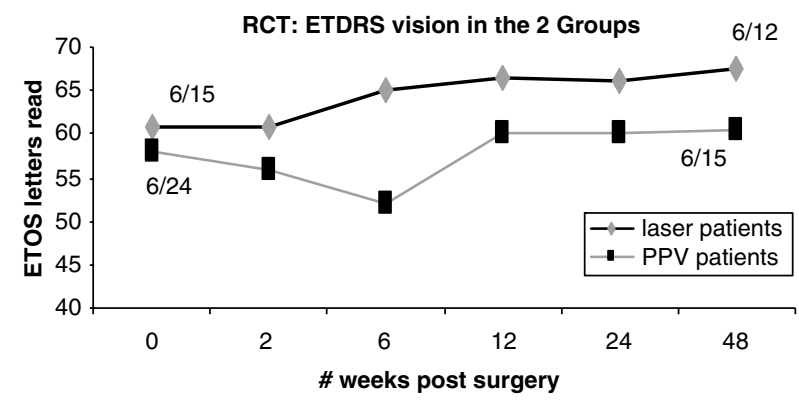

Figure 2 Comparing ETDRS vision between the two groups after treatment.

Table 2 Final functional and structural results 12 months after intervention for each treatment

\begin{tabular}{lccccc}
\hline Median values & \multicolumn{2}{c}{ Laser } & & \multicolumn{2}{c}{ Vitrectomy } \\
\cline { 2 - 3 } \cline { 5 - 6 } & Baseline & 12 months & & Baseline & 12 months \\
\hline ETDRS letters & 62.5 & 67.5 & & 58 & 60 \\
Snellen VA & $6 / 15$ & $6 / 12$ & & $6 / 24$ & $6 / 15$ \\
Foveal thickness & 390 & 283 & & 364 & 337 \\
Macular volume & 3.55 & 2.77 & & 3.23 & 2.81 \\
\hline
\end{tabular}

The data were analysed data as percentage success rate (defined as either an improvement or stability with no loss of visual acuity greater than one line) at each time point compared to baseline. For the laser group, it was $88 \%(6 / 8)$ at 6 weeks, $75 \%$ at 12 weeks, and then $100 \%$ at 6 months with a final success percentage at 12 months postlaser treatment was $75 \%$ (6/8). Similarly, for the PPV group success percentage were $57 \%$ at 6 weeks, and $86 \%$ both at 12 and 24 weeks with a final success percentage of $71 \%$ at 12 months. This showed that the proportion of patients with successful ETDRS acuity outcome after laser photocoagulation was $4 \%$ greater than after PPV.

\section{OCT results}

The median foveal thickness in the laser group at baseline was $390.5 \mu \mathrm{m}$ (range 191-666 $\mu \mathrm{m}$ ) and remained at a similar level $(395.5 \mu \mathrm{m}$, range $195-636 \mu \mathrm{m})$ at 6 weeks post-treatment. During the remaining follow-up period, the foveal thickness gradually decreased from the baseline: at 3 months, it was $298.5 \mu \mathrm{m}$ (range 186-424 $\mu \mathrm{m}$ ), while at 6 months, it was $318 \mu \mathrm{m}$ (range 184-550 $\mu \mathrm{m})$, and at 12 months, it was $283 \mu \mathrm{m}$ (range $173-480 \mu \mathrm{m}$ ) (Figure 3 ). The macular volume and its change over time reflected the changes in the foveal thickness. The median baseline volume in this group was $3.55 \mathrm{~mm}^{3}$ (range $2.08-5.75 \mathrm{~mm}^{3}$ ), which showed a gradual decrease over the follow-up period. By 6 weeks it was $3.48 \mathrm{~mm}^{3}$ (range $2.33-5.89 \mathrm{~mm}^{3}$ ), at 3 months, it was $2.92 \mathrm{~mm}^{3}$ (range 2.34-4.09 $\mathrm{mm}^{3}$ ), and at 6 months $3.3 \mathrm{~mm}^{3}$ (range $2.14-4.18 \mathrm{~mm}^{3}$ ) with a final decrease to $2.77 \mathrm{~mm}^{3}$ (range 2.16-4.49 $\mathrm{mm}^{3}$ ) (Figures 4 and 5; Table 2).

The median baseline foveal thickness in the PPV group was $364 \mu \mathrm{m}$ (range 198-607 $\mu \mathrm{m}$ ), which showed a gradual decrease over the 12-month postoperative period. At 6 weeks, it was $337 \mu \mathrm{m}$ (range 197-501 $\mu \mathrm{m}$ ), at 3 months, it was $342 \mu \mathrm{m}$ (range $230-424 \mu \mathrm{m}$ ), at 6 months, it was $276.5 \mu \mathrm{m}$ (range 192-501 $\mu \mathrm{m}$ ), and at 12 months, it had decreased to $337 \mu \mathrm{m}$ (range 183-467 $\mu \mathrm{m}$ ) (Figure 3).

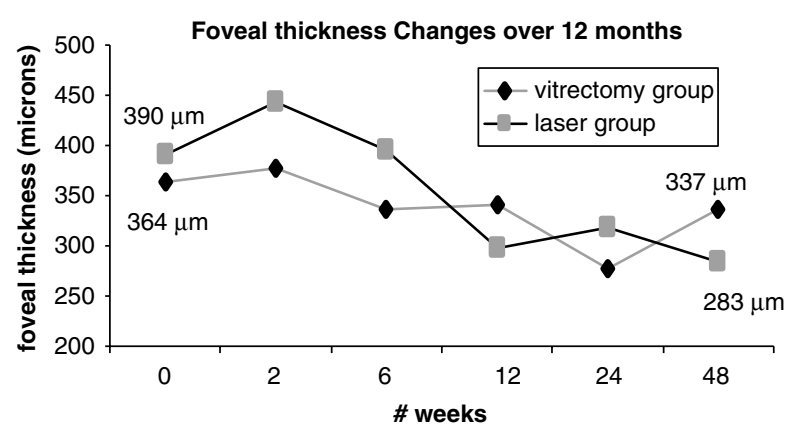

Figure 3 Comparing foveal thickness between the two groups after treatment. 
Similarly, the median baseline volume was $3.23 \mathrm{~mm}^{3}$ (range 2.26-4.13 $\mathrm{mm}^{3}$ ), which then decreased to $3.04 \mathrm{~mm}^{3}$ (range $2.34-3.65 \mathrm{~mm}^{3}$ ) at 6 weeks, $2.8 \mathrm{~mm}^{3}$ (range $2.42-$ $\left.3.44 \mathrm{~mm}^{3}\right)$ at 3 months, $2.46 \mathrm{~mm}^{3}\left(1.8-3.55 \mathrm{~mm}^{3}\right)$ at 6 months, and then at 12 months it had showed a small increase to $2.81 \mathrm{~mm}^{3}$ (range $2.25-3.97 \mathrm{~mm}^{3}$ ) (Figures 4 and 6) (illustrates OCT macula changes seen in the vitrectomy group; Table 2).

Comparing the changes in ETDRS vision in the two groups over time, the laser group showed a greater level of improvement at each time point compared to the PPV group (Figure 7). Similar assessment of changes in the foveal thickness and macular volume confirmed that the laser group showed a greater level of improvement at

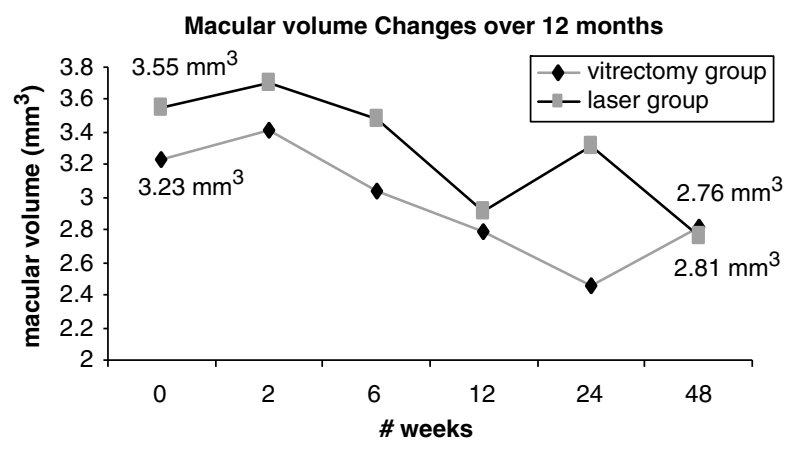

Figure 4 Comparing macular volume between the two groups after treatment. each follow-up visit compared to the PPV group (Figure 8).

The preoperative macular profiles on OCT revealed only one patient in each group having had the domeshaped pattern of macular thickening with an attached posterior hyaloid face only at the foveal tip. All the other patients had the diffuse-low thickening of the macula with no signs of focal posterior hyaloid separation. The one patient in the PPV group with the dome-shaped profile showed a decreasing foveal thickness and macular volume over the 1-year postoperative follow-up period, so that by 12 months the foveal thickness was $385 \mu \mathrm{m}$ (baseline $472 \mu \mathrm{m}$ ) and the macular volume was $3.18 \mathrm{~mm}^{3}$ (baseline $3.74 \mathrm{~mm}^{3}$ ). Reflecting this structural improvement, both the ETDRS vision and the Snellen acuity showed improvements: ETDRS vision improved from 53 letters read at baseline to 63 letters read at 12 months or a baseline corrected Snellen of 20/80 to 12 months Snellen of 20/50. In the laser patient with domeshaped macular thickening, the foveal thickness remained high despite laser treatment in the early followup period, but by 6 and 12 months post-treatment, both these structural indices showed improvements. At 12 months, foveal thickness was $207 \mu \mathrm{m}$ (baseline $666 \mu \mathrm{m}$ ) and the macular volume was $2.28 \mathrm{~mm}^{3}$ (baseline $5.38 \mathrm{~mm}^{3}$ ). However, the visual acuity remained stable at 20/50. Serial OCT in this patient revealed evidence of spontaneous posterior vitreous separation 3 months after

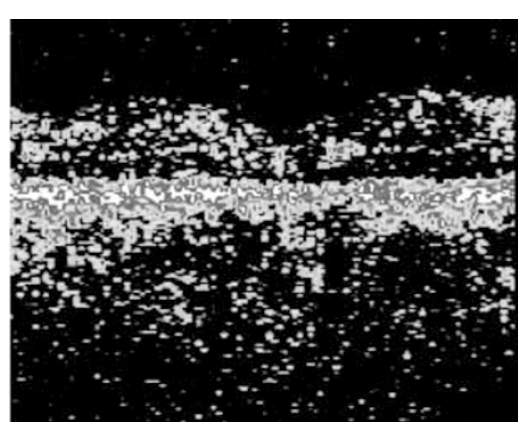

Pre op: $191 \mu \mathrm{m} / 2.08 \mathrm{~mm}^{3}$

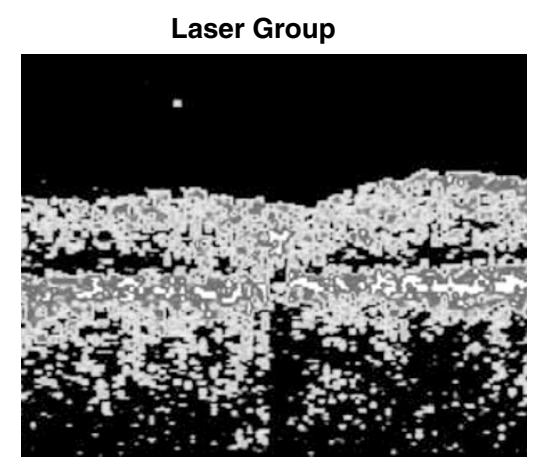

6 weeks: $195 \mu \mathrm{m} / 2.33 \mathrm{~mm}^{3}$

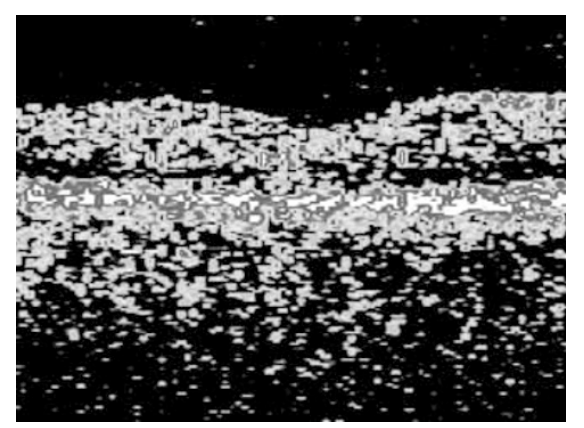

3 months: $186 \mu \mathrm{m} / 2.34 \mathrm{~mm}^{3}$

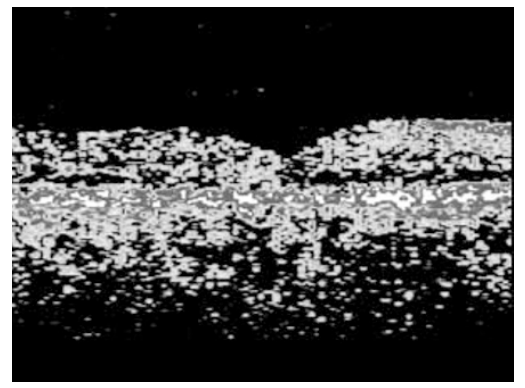

6 months: $184 \mu \mathrm{m} / 2.14 \mathrm{~mm}^{3}$

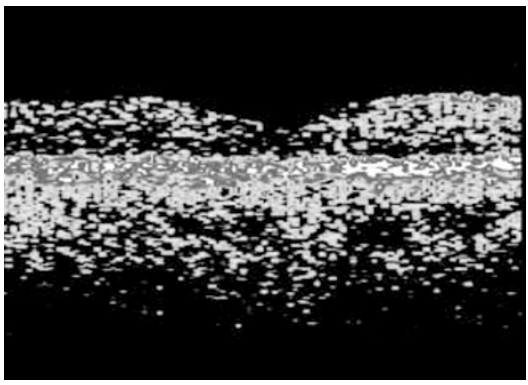

12 months: $173 \mu \mathrm{m} / 2.16 \mathrm{~mm}^{3}$

Figure 5 Macula OCT changes for a patient in the Laser Group after treatment. 


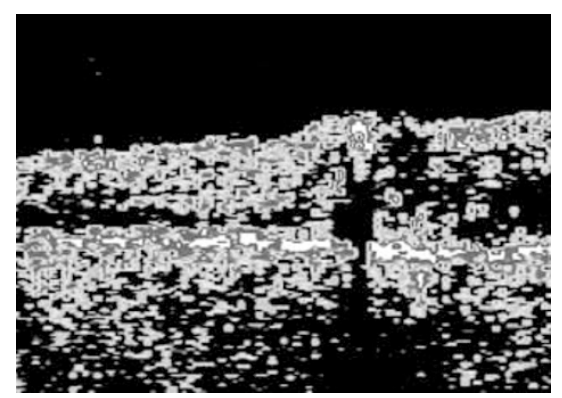

Pre op: $265 \mu \mathrm{m} / 2.59 \mathrm{~mm}^{3}$

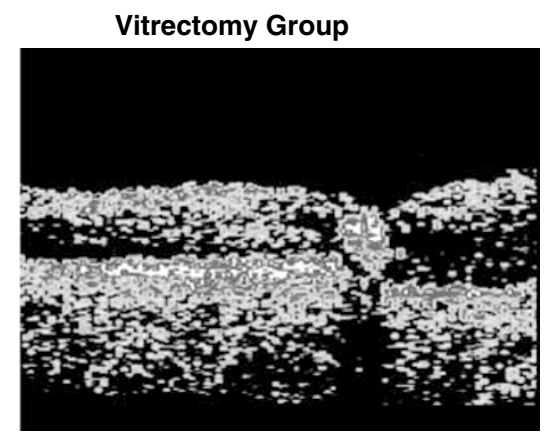

6 weeks: $316 \mu \mathrm{m} / 2.99 \mathrm{~mm}^{3}$

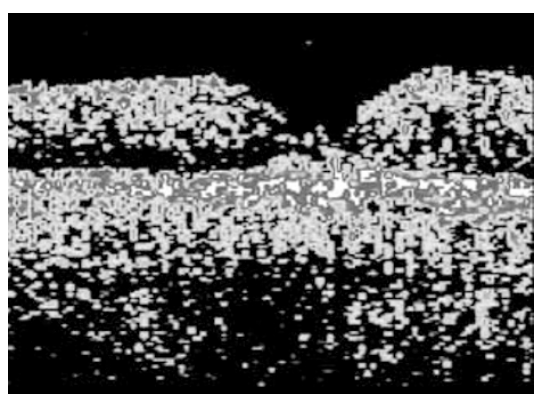

3 months: $230 \mu \mathrm{m} / 2.42 \mathrm{~mm}^{3}$

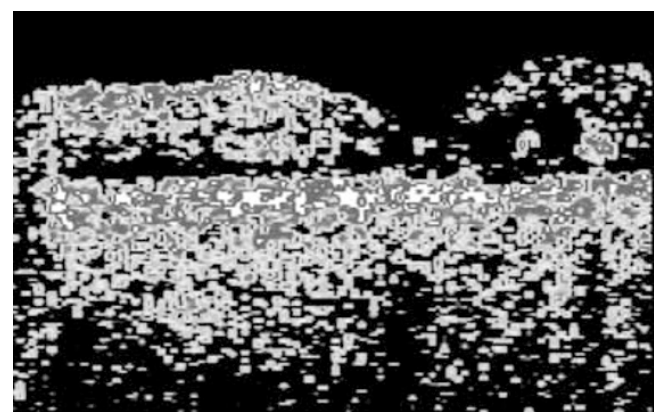

6 months: $269 \mu \mathrm{m} / 2.53 \mathrm{~mm}^{3}$

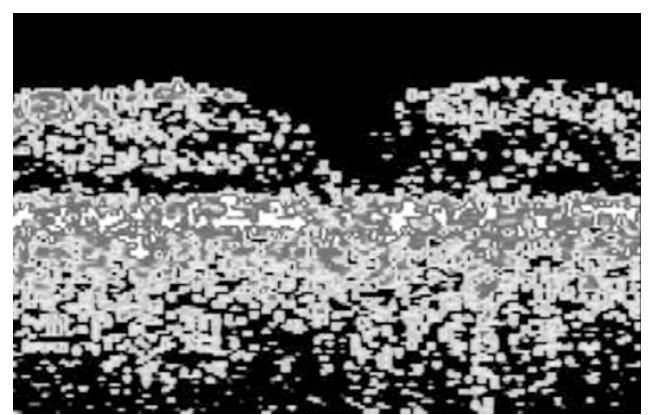

12 months: $183 \mu \mathrm{m} / 2.39 \mathrm{~mm}^{3}$

Figure 6 Macula OCT changes for a patient in the Vitrectomy Group after treatment.

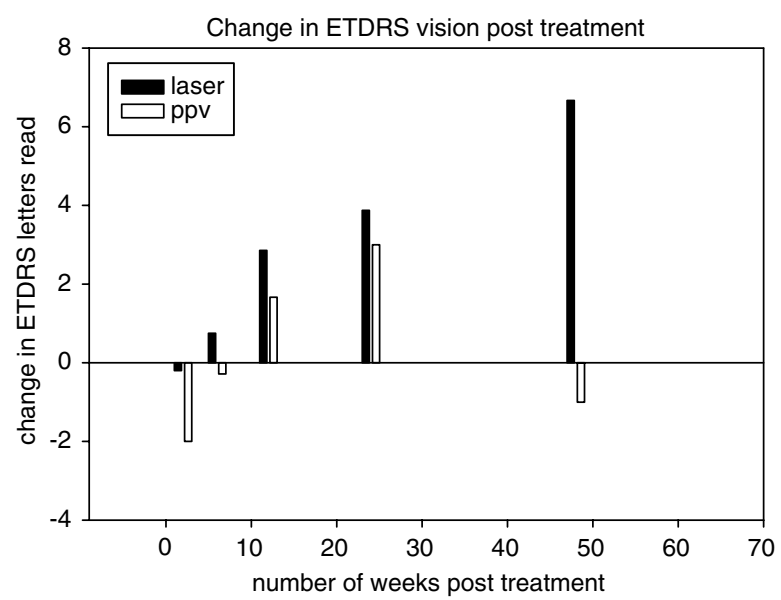

Figure 7 Comparing the changes in ETDRS vision between the two groups after treatment.

treatment. Two other patients in the laser group had evidence of similar OCT-confirmed spontaneous vitreous detachment during the 1-year follow-up period with similar improvement in both the macula structural indices and visual acuity (Figure 9).

\section{FMM results}

The log thresholds of the perifoveal cones showed little difference between the two groups after treatment despite both treatments individually showing improvement in the cone function. Comparing the change in cone thresholds over time, the PPV group showed a greater level of perifoveal functional improvement compared to the laser group (Figure 10). Individually only one patient in the laser-treated arm showed a nonsignificant but modest improvement in the perifoveal cone thresholds after macular laser treatment. The other patients in this treated group showed a small worsening in the perifoveal cone function.

\section{Discussion}

The treatment of diffuse macular oedema is notoriously difficult with limited success in improving vision. Focal laser treatment can stabilize vision and has become the standard treatment for clinically significant diabetic macular oedema. ${ }^{1}$ In contrast, published studies to date have suggested positive results in macular function and structure after PPV. However, many of these studies are retrospective, had small number of cases or lacked standardised protocols for visual acuity determination, and most importantly lacked a randomised control group. One prospective study did suggest improvement in the macular oedema but not in the vision at the end of the follow-up period in a group of seven patients with macular oedema present for $<1$ year. $^{9}$ 

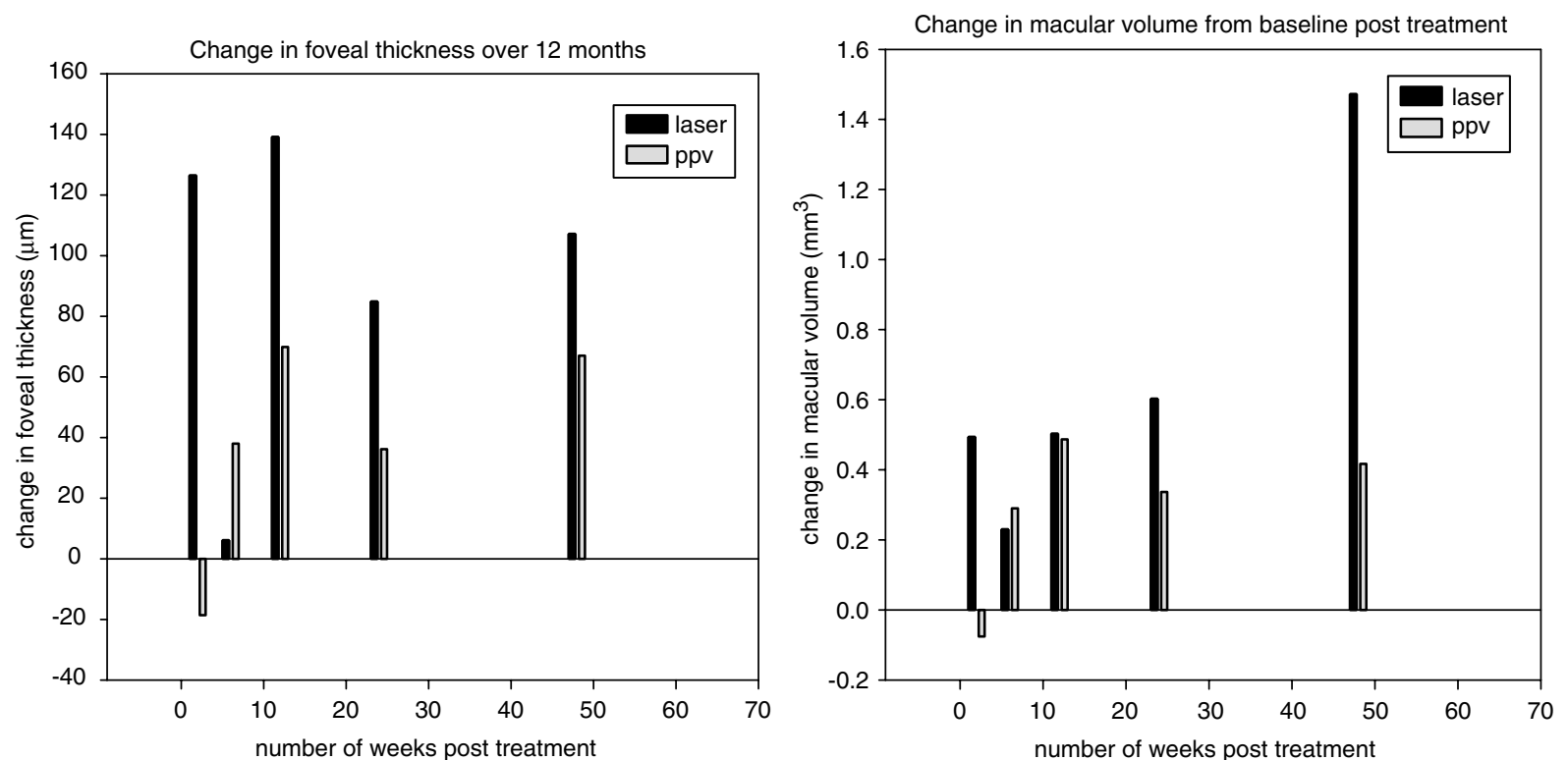

Figure 8 Comparing the structural macular changes between the two groups after treatment.

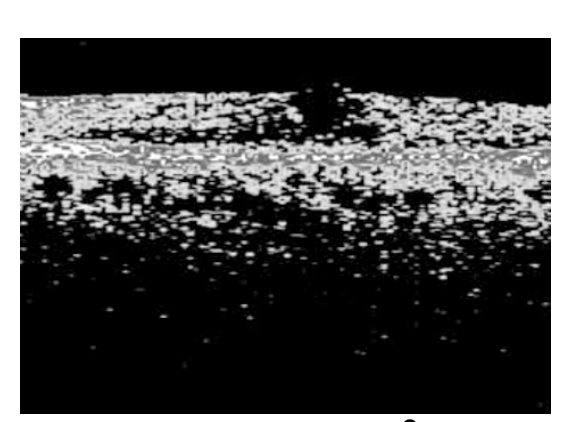

Pre op: $322 \mu \mathrm{m} / 2.72 \mathrm{~mm}^{3}$

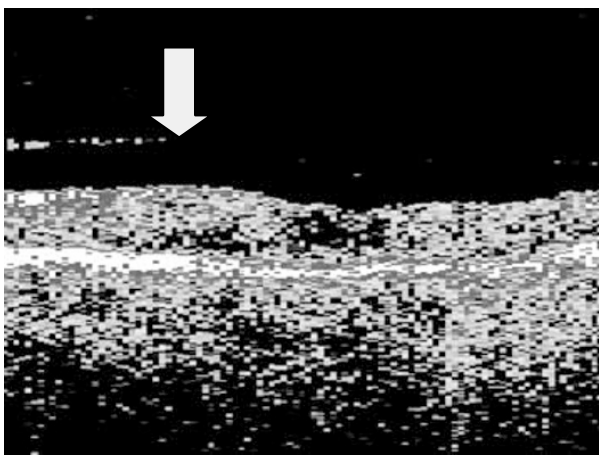

6 months: $245 \mu \mathrm{m} / 2.37 \mathrm{~mm}^{3}$

Arrow illustrates position of the detached posterior hyaloid face

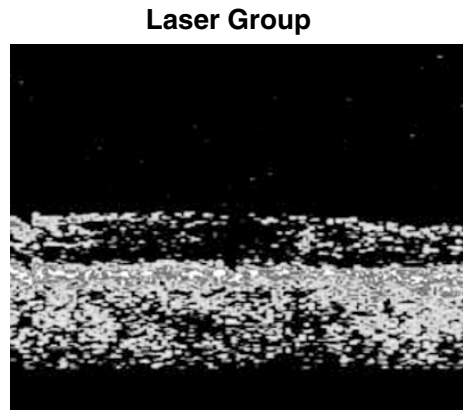

6 weeks: $268 \mu \mathrm{m} / 2.42 \mathrm{~mm}^{3}$

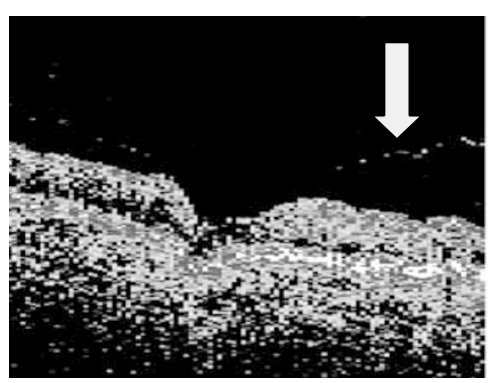

3 months: $268 \mu \mathrm{m} / 2.4 \mathrm{~mm}^{3}$

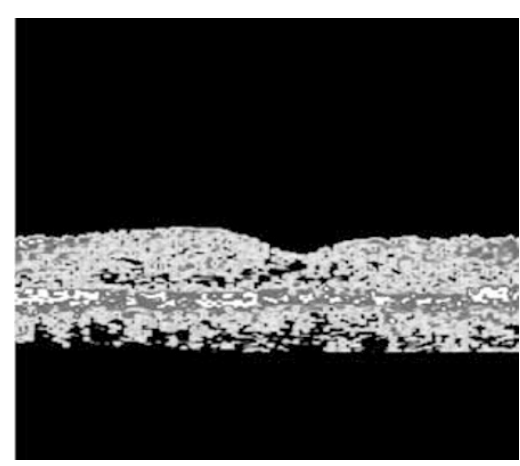

12 months: $192 \mu \mathrm{m} / 2.19 \mathrm{~mm}^{3}$

Arrow illustrates position of the spontaneous posterior hyaloid detachment

Figure 9 OCT captured spontaneous posterior vitreous detachment in a Laser Group patient.

Eventhough this pilot randomised trial of laser photocoagulation against PPV for diabetic macular oedema was designed to assess methods and estimate sample size for a larger definitive study, its results do provide an initial assessment of the role of PPV in diabetic macular oedema as part of a controlled randomised trial.

The findings from this pilot trial suggest no advantage of PPV over conventional ETDRS laser in the management of diabetic macular oedema. However, we 


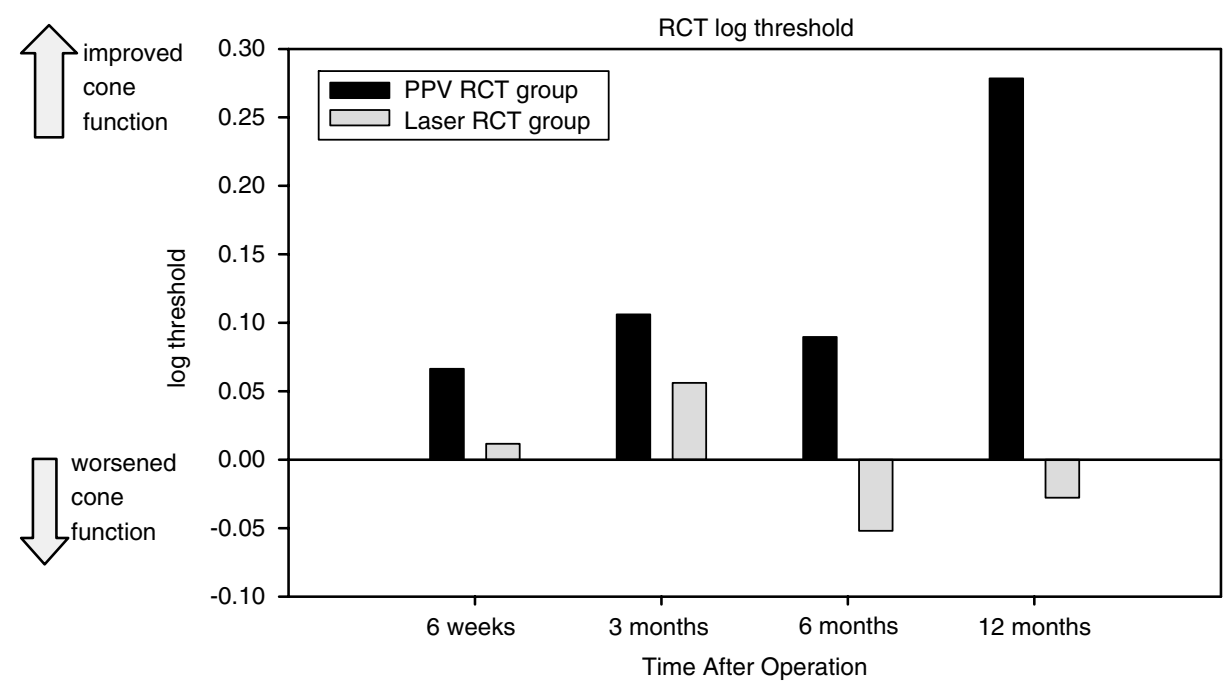

Figure 10 Comparing the changes in the cone function between the two groups after treatment.

also demonstrated a stabilisation of ETDRS vision in four of the PPV patients with improvement in the vision in one of them. The PPV group showed a greater improvement in the perifoveal cone function over the 12-month follow-up period.

A combination of vasopermeability-inducing cytokines as well as vitreomacular tractional forces is thought to promote the development of diabetic macular oedema. Alleviation of such forces by PPV would allow for resolution of macular oedema. ${ }^{4}$ The patients included in the trial all had persistent CSME present for at least 12 months in spite of prior laser photocoagulation with an average of three macular laser treatments. The OCT macular profiles at baseline suggested only one patient in each group having had the dome-shaped macular configuration with partial PVD and vitreofoveal attachment. It is conceivable that tractional forces in such cases may significantly contribute to the pathogenesis of macular oedema. The others had the diffuse-low elevation profile where no such vitreomacular relationship was seen on OCT.

Four patients from both groups showed structural and functional improvement 12 months after initial treatment. One patient belonged to the vitrectomy group, while the other three, belonging to the laser group, developed spontaneous vitreous detachment 3 months after laser treatment.

This suggests that separation of the posterior vitreous cortex from the macular surface either by vitrectomy or as a spontaneous event may allow for structural and functional improvement which is variable. This variability may reflect the continuing actions of the vasopermeability-inducing cytokines produced in diabetes and/or a possible damage to the macular surface by the peeling of the posterior hyaloid. The histological examination of membranes harvested at vitrectomy from patients with vitreomacular traction syndrome revealed large segments of the ILM. ${ }^{10}$ This is in contrast to plasmin-induced vitreoretinal separation, which resemble more closely spontaneous PVD, no such damage to the ILM was seen. ${ }^{11}$ Damage to the ILM may change Muller cell physiology and so alter electrophysiological recovery as tested by ERG. ${ }^{12}$ The less traumatic spontaneous PVD that occurred in three patients in the laser arm may have contributed to the thinning of the macula and to the visual improvement and thus narrowed the difference in the success rates for each treatment arm at 12 months. Also, macular function as measured electrophysiologically is compromised in diabetic patients with macular oedema after focal laser treatment. ${ }^{13-15}$ This compromise seems to involve the loss of foveal cones, ${ }^{14}$ oedema-induced changes in Muller cells, ${ }^{16,17}$ as well as the associated damage of nontreated areas of adjacent retina. ${ }^{13}$ In our series, the patients were recruited after having undergone previous macular laser treatment (an average of three times) with the oedema present for an average of 14 months prior to treatment in each arm. Both the high number of laser treatments and the relatively long duration of oedema may have contributed to the limited improvement in foveal cone function.

However, the improvement after vitrectomy may have also been in part due to the removal of the vasopermeability-inducing growth factors contained in the vitreous gel (eg vascular endothelial growth factor (VEGF)) and the improved oxygenation of the retina postvitrectomy, ${ }^{18}$ which would allow for a decreased VEGF production. 
Intravitreal triamcinolone or ILM peel were not included as part of the treatment regimen because we felt this would add another variable and that ILM peel was to be introduced as part of a separate pilot study.

The results of this pilot trial suggest little difference between the two treatments, that is, that the proportion of eyes with a successful visual acuity outcome after laser photocoagulation was $4 \%$ greater than after PPV (75 vs $71 \%$ ). This percentage success rate is an estimate based on the patients seen at the 12 months follow-up time point. Two patients in the laser group and one in the vitrectomy group failed attendance at this final time point and so no assumption was made as to whether they were successful or a failure in terms of ETDRS vision. The aim of the study was to determine the numbers needed for a definitive study in this setting. Given the small numbers and the drop-out rate (as high as 25\%), the following calculations can be made: to detect (at $5 \%$ significance) a relative improvement $4 \%$ at 12 months would require approximately 4647 patients per treatment arm (9294 total) (90\% power). Given this small difference between the treatment groups, the very large numbers needed for a definitive randomised trial and that our data revealed a small but significant improvement in vision in the laser-treated patients, such a study may prove to be an organisational challenge as it would require participation of a large number of centres.

\section{References}

1 The Early Treatment Diabetic Retinopathy Study Research Group. Photocoagulation for diabetic macular edema. Early Treatment Diabetic Retinopathy Study Report No. 1. Arch Ophthalmol 1985; 103: 1796-1806.

2 Lee C, Olk R. Modified grid laser photocoagulation for diffuse diabetic macular edema: long term visual results. Ophthalmology 1991; 98: 1594-1602.

3 Bresnick G. Diabetic maculopathy. A critical review highlighting diffuse macular edema. Ophthalmology 1983; 90: 1301-1317.

4 Lewis H, Abrams G, Blumenkranz M, Campo R. Vitrectomy for diabetic macular traction edema associated with posterior hyaloidal traction. Ophthalmology 1992; 99: 753-759.
5 Heij E, Hendrikse F, Kessels A, Derhaag P. Vitrectomy results in diabetic macular oedema without evident vitreomacular traction. Graefe's Arch Clin Exp Ophthalmol 2001; 239: 264-270.

6 Duguid G, Massin P, Haouchine B, Erginay A, Gaudric A. Vitrectomy for diabetic macular oedema: the effect on visual acuity and optical coherence tomography (OCT) appearances. Invest Ophthalmol Vis Sci 2000; 41: 585.

7 Giovannini A, Amato G, Mariotti C, Scassellati-Sforzolini B. Optical Coherence Tomography findings in diabetic macular edema before and after vitrectomy. Ophthalmic Surg Lasers 2000; 31: 187-191.

8 Huang D, Swanson E, Lin C, Schuman JS, Stinson WG, Chang W et al. Optical coherence tomography. Science 1991; 254: 1178-1181.

9 Otani T, Kishi S. A controlled study of vitrectomy for diabetic macular edema. Am J Ophtalmol 2002; 134: 214-219.

10 Shinoda K, Hirakata A, Hida T, Yamaguchi Y, Fukuda M, Maekawa S. et al.Ultrastructural and immunohistochemical findings in five patients with vitreomacular traction syndrome. Retina 2000; 20: 289-293.

11 Gandofer A, Messmer E, Ulbig M, Kampik A. Resolution of diabetic macular edema after surgical removal of the posterior hyaloid and inner limiting membrane. Retina 2000; 20: 126-133.

12 Terasaki H, Miyake Y, Nomura R, Piao Chhori K, Niwa T, Kondo M. Focal macular ERGs in eyes after removal of macular ILM during macular hole surgery. Invest Ophthalmol Vis Sci 2001; 42: 229-234.

13 Greenstein V, Holopigian K, Hood D, Seiple W, Carr R. The nature and extent of retinal dysfunction associated with diabetic macular edema. Invest Ophthalmol Vis Sci 2000; 41: 3643-3654.

14 Lardenoye C, Probst K, DeLint P, Rothova A. Photoreceptor function in eyes with macular edema. Invest Ophthalmol Vis Sci 2000; 41: 4048-4053.

15 Greenstein V, Chen H, Hood D, Holopigian K, Seiple W, Carr R. Retinal function in diabetic macular edema after focal laser photocoagulation. Invest Ophthalmol Vis Sci 2000; 41: 3655-3664.

16 Yamamoto S, Yamamoto T, Hayashi M, Takeuchi S. Morphological and functional analyses of diabetic macular edema by optical coherence tomography and multifocal electroretinograms. Graefe's Arch Clin Exp Ophthalmol 2001; 239: 96-101.

17 Fine B, Brucker A. Macualr edema and cystoid macular edema. Am J Ophthalmology 1981; 92: 466-481.

18 Stefansson H-L. The therapeutic effects of retinal laser treatment and vitrectomy. A theory based on oxygen and vascular physiology. Acta Ophthalmol Scand 2001; 79: 435-440. 